\title{
Synthesis and Anti-HIV Activity of Novel 4'-Ethyl-5'-norcarbocyclic Adenosine Phosphonic Acid Analogues
}

\author{
Jin Cheol Yoo, Hua Li, Wonjae Lee, and Joon Hee Hong* \\ BK-21 Project Team, College of Pharmacy, Chosun University, Kwangju 501-759, Korea \\ *E-mail:hongjh@chosun.ac.kr
}

Received July 20, 2010, Accepted September 15, 2010

\begin{abstract}
Novel 4'-ethyl-5'-norcarbocyclic adenosine phosphonic acid analogues were synthesized from propionaldehyde $\mathbf{5}$ through a de novo acyclic synthetic route using reiterative Grignard additions and ring-closing metathesis (RCM) as key reactions. The synthesized nucleoside phosphonic acids analogues 17, 18, 19, and 21 were subjected to antiviral screening against human immunodeficiency virus.
\end{abstract}

Key Words: Anti-HIV agents, 4'-Ethyl branched nucleoside, Phosphonic acid nucleosides

\section{Introduction}

4'-Branched nucleosides were first investigated by Maag et al. ${ }^{1}$ in 1992, and 4'-azido-thymidine (1) exerts potent activity against HIV-1. Extensive structure-activity studies found that other 4'-position lipophilic substituents, such as 4'-ethylthymidine (2), ${ }^{2}$ also exhibited high antiviral activity against HIV. Molecular modeling studies demonstrated the presence of a narrow, relatively hydrophobic 4'-pocket that can accommodate these substitutions, contributing to enhanced potency. ${ }^{3}$

5 '-Nornucleoside phosphonic acid analogues such as d4AP $(3)^{4}$ may be potential anti-HIV agents and have encouraged the search for novel nucleosides in this class of compounds. ${ }^{5}$ The spatial location of the oxygen atom, namely the $\beta$-position from the phosphorus atom in the nucleoside analogue, plays a critical role for antiviral activity by increasing binding capacity of the phosphonate analogues to target enzymes. ${ }^{6}$ The phosphonate has certain advantages over its phosphate counterpart as it is metabolically stable because its phosphorus-carbon bond is not susceptible to hydrolytic cleavage. ${ }^{7}$ Moreover, a phosphonate nucleoside analogue can skip the requisite first phosphorylation, a rate-limiting crucial step for the activation of nucleosides that ultimately leads to triphosphates. ${ }^{8}$

Carbocyclic nucleosides are a group of compounds structurally similar to natural nucleosides in which the furanose oxygen is replaced by a methylene group. Replacement of the furanose ring oxygen by carbon is of particular interest because the resulting carbocyclic nucleosides possess greater metabolic stability to phosphorylase, ${ }^{9}$ which cleaves the glycosidic bond of nucleosides. The recent discovery of 4'-ethynyl-cpAP $(4)^{3}$ as an anti-HIV agent gave strong impetus to the search for novel nucleosides in this class of compounds.

Stimulated by these findings that 4'-branched nucleoside analogues and 5'-nornucleoside phosphate had excellent biological activities, we sought to synthesize a novel class of nucleosides comprising 4'-ethyl branched carbocyclic-5'-norcarbocyclic phosphonic acid analogues to search for more efficient therapeutic agents against HIV and to provide analogues used in probing the conformational preferences of enzymes associated with the metabolism of nucleosides and nucleotides.

\section{Results and Discussions}

As depicted in Scheme 1, the target compounds were prepared from protected propionaldehyde $\mathbf{5}$. $^{10}$ The aldehyde functional group of $\mathbf{5}$ was subjected to a carbonyl addition reaction by ethylmagnesium bromide to furnish the secondary alcohol $\mathbf{6}$, which was subjected to oxidation using pyridium chlorochromate (PCC) to provide ketone derivative 7 . The corresponding ketone functional group of 7 was again subjected to an addition reaction by vinylmagnesium bromide to give the tertiary hydroxyl analogue $( \pm)-8$, which was successfully protected using $p$-methoxybenzyl chloride (PMBCl) to give the fully protected compound $( \pm)-9$. Removal of the silyl protecting group of $( \pm)-9$ using $t$-butylammonium fluoride (TBAF) provided the primary alcohol $( \pm)$-10, which was oxidized to the aldehyde $( \pm)$-11 using Swern oxidation conditions (DMSO, oxalyl chloride, TEA). The aldehyde $( \pm)$-11 was subjected to nucleophilic Grignard conditions with vinylmagnesium bromide to give divinyl $( \pm)-\mathbf{1 2}$, which was subjected to ring-closing metathesis (RCM) conditions using a 2 nd generation Grubbs catalyst ${ }^{11}$ to provide ethyl-

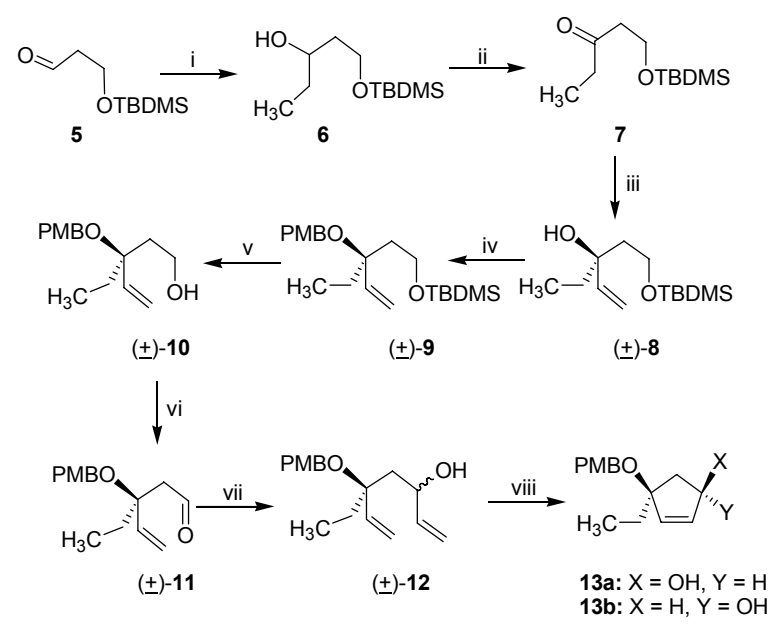

Reagents: i) ethylMgBr, THF; ii) PCC, $\mathrm{CH}_{2} \mathrm{Cl}_{2}$; iii) vinylMgBr, THF; iv) PMBCl, $\mathrm{NaH}$, DMF; v) TBAF, THF; vi) $(\mathrm{COCl})_{2}$, DMSO, TEA; vii) vinylMgBr, THF; viii) Grubbs (II), $\mathrm{CH}_{2} \mathrm{Cl}_{2}$.

Scheme 1. Synthesis of key cyclopentenol intermediate 13b 
<smiles>Cc1cn(C2COC2(N)CO)c(=O)[nH]c1=O</smiles>

4'-azidothymidine (1)

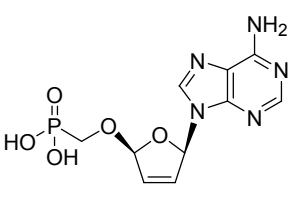

d4AP (3)

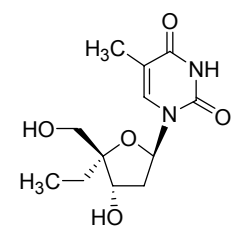

4'-ethylthymidine (2)

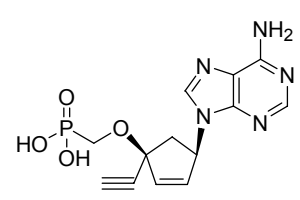

4'-ethynyl-cpAP (4)
Figure 1. Some structures of nucleoside analogues as potent anti-HIV agents.

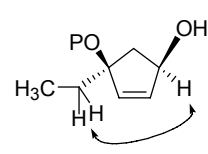

13a $(0.82 \%)$

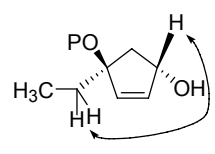

$13 b(0.24 \%)$
Figure 2. NOE differences between the proximal hydrogens of 13a and $\mathbf{1 3 b}$.

ated cyclopentenol 13a (39\%) and $\mathbf{1 3 b}(40 \%)$, which were readily separated by silica gel column chromatography. The NOE experiments with cyclpopentenols 13a and 13b confirmed these assignments. As expected, NOE enhancements were found between the cis-oriented hydrogens. Upon irradiation of $C_{1}-\mathrm{H}$, weak NOE patterns were observed at the proximal hydrogens of compound $\mathbf{1 3 b}\left[\mathrm{C}_{4}-\mathrm{CH}_{2}(0.24 \%)\right]$ versus those of compound $13 \mathrm{a}\left[\mathrm{C}_{4}-\mathrm{CH}_{2}(0.82 \%)\right]$ (Figure 2$)$.

To synthesize the desired 5'-norcarbocyclic adenine nucleoside analogue, the protected cyclopentenol 13b was treated with 6-chloropurine under Mitsunobu conditions (DIAD and $\mathrm{PPh}_{3}$ ). Slow addition of diisopropyl azodicarboxylate (DIAD) to a mixture of cyclopentenol 13b, triphenylphosphine, and the 6-chloropurine in anhydrous tetrahydrofuran gave a yellow solution that was stirred for $3 \mathrm{~h}$ at $-20^{\circ} \mathrm{C}$ to give a protected 6-chloropurine analogue 14 (Scheme 2). ${ }^{12}$ The PMB protection group was removed with 2,3-dichloro-5,6-dicyano- $p$-benzoquinone (DDQ) ${ }^{13}$ to the 5'-nornucleoside analogue $\mathbf{1 5}$, which was treated with diethylphosphonomethyl triflate ${ }^{14}$ using lithium $t$-butoxide to yield the nucleoside phosphonate 16. The chlorine group of $\mathbf{1 6}$ was then converted to amine with methanolic ammonia at $70{ }^{\circ} \mathrm{C}$ to give a corresponding adenine phosphonate derivative $\mathbf{1 7}$.

The nucleoside phosphonate mimics the overall shape and geometry of a nucleoside monophosphate. Hydrolysis of 17 by treatment with bromotrimethylsilane in $\mathrm{CH}_{3} \mathrm{CN}$ in the presence of 2,6-lutidine gave an adenine phosphonic acid derivative $\mathbf{1 8} .^{15}$

Bishydroxylation ${ }^{16}$ of the double bond in 17 was accomplished with a catalytic amount of osmium tetraoxide $\left(\mathrm{OsO}_{4}\right)$ and 4-methyl-morpholine $\mathrm{N}$-oxide (NMO) as the oxidant to give the dihydroxylated isomer $\mathbf{1 9}(34 \%)$ and $\mathbf{2 0}(29 \%)$, with a relatively low stereoselectivity (Scheme 3). ${ }^{17}$ Their stereochemistries were also readily determined by NOE experiments. These stereochemical outcomes suggest that the steric environments of $\alpha$ and

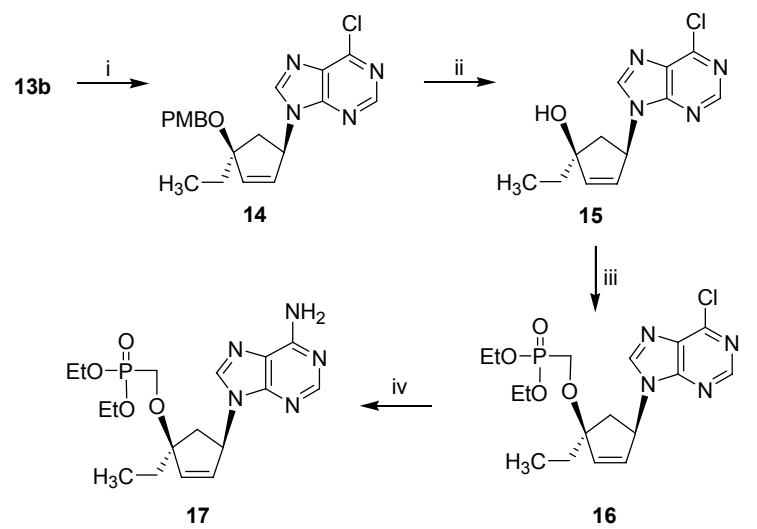

Reagents: i) 6-chloropurine, DIAD, THF; ii) DDQ, $\mathrm{CH}_{2} \mathrm{Cl}_{2} / \mathrm{H}_{2} \mathrm{O}$, rt;; iii) (EtO) ${ }_{2} \mathrm{POCH}_{2} \mathrm{OTf}$, LiO-t-Bu, THF; iv) $\mathrm{NH}_{3} / \mathrm{MeOH}, 70^{\circ} \mathrm{C}$.

Scheme 2. Synthesis of 5'-norcarbocyclic adenosine phosphonate

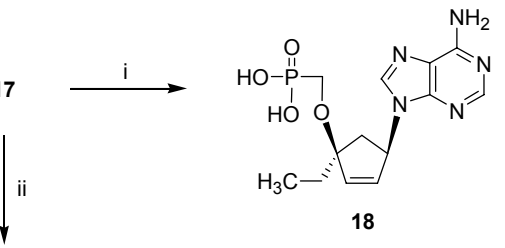<smiles>[R6][R]([R6])([H])CO[C@]1(CC)C[C@@H](n2cnc3c(N)ncnc32)[C@H](O)[C@@H]1O</smiles>

$$
\text { i 21: } \mathrm{R}=\mathrm{H}
$$

Reagents: i) TMSBr, 2,6-lutidine, $\mathrm{CH}_{3} \mathrm{CN}$; ii) $\mathrm{OsO}_{4}, \mathrm{NMO}$ acetone $/ \mathrm{t}-\mathrm{BuOH} / \mathrm{H}_{2} \mathrm{O}$.

Scheme 3. Synthesis of desired 5'-norcarbocyclic adenosine phosphonic acid analogues

Table 1. Anti-HIV activity of synthesized compounds

\begin{tabular}{ccc}
\hline Compound No. & $\begin{array}{c}\text { anti-HIV-1 } \\
\mathrm{EC}_{50}(\mu \mathrm{M})^{b}\end{array}$ & $\begin{array}{c}\text { Cytotoxicity } \\
\mathrm{CC}_{50}(\mu \mathrm{M})^{c}\end{array}$ \\
\hline $\mathbf{1 7}$ & 55 & 95 \\
$\mathbf{1 8}$ & 21 & 58 \\
$\mathbf{1 9}$ & $>100$ & $>100$ \\
$\mathbf{2 1}$ & $>100$ & $>100$ \\
PMPA $^{a}$ & 3.6 & $>100$ \\
\hline
\end{tabular}

${ }^{a}$ PMPA: 9-(2-phosphonylmethoxypropyl)adenine. ${ }^{b} \mathrm{EC}_{50}(\mu \mathrm{M})$ : Concentration $(\mu \mathrm{M})$ required to inhibit the replication of $\mathrm{HIV}-1$ by $50 \% .{ }^{c} \mathrm{CC}_{50}$ $(\mu \mathrm{M})$ : Concentration $(\mu \mathrm{M})$ required to reduce the viability of unaffected cells by $50 \%$.

$\beta$-faces on the cyclopentene ring might be equivalent. Hydrolysis of the diethyl phosphonate functional groups of $\mathbf{1 9}$ by a similar procedure described for $\mathbf{1 8}$ gave an adenine phosphonic acid derivative 21 .

The synthesized nucleoside phosphonate and phosphonic acid analogues 17, 18, 19, and 21 were evaluated for antiviral activity against HIV-1.

As shown in Table 1, nucleoside phosphonic acid 18 exhibited greater toxicity-dependent anti-HIV activity than its parent 
nucleoside phosphonate 17. However, nucleotide analogs 19 and $\mathbf{2 1}$ did not show anti-HIV activity nor cytotoxicity up to $100 \mu \mathrm{M}$.

In summary, on the basis of potent anti-HIV activity of 4'alkyl branched nucleoside and 5'-norcarbocyclic nucleoside analogues, we have designed and successfully synthesized novel 4'-ethyl-5'-norcarbocyclic nucleotide analogues starting from propionaldehyde. In this series, adenosine phosphonic acid derivative 18 showed moderate toxicity-dependent anti-HIV-1 activity.

\section{Experimental Section}

Melting points were determined on a Mel-temp II laboratory device and are uncorrected. NMR spectra were recorded on a JEOL 300 Fourier transform spectrometer (JEOL, Tokyo, Japan); chemical shifts are reported in parts per million $(\delta)$ and signals are reported as $\mathrm{s}$ ( singlet), d (doublet), t (triplet), q (quartet), $m$ (multiplet), and dd (doublet of doublets). UV spectra were obtained on a Beckman DU-7 spectrophotometer (Beckman, South Pasadena, CA, USA). MS spectra were collected in electrospray ionization (ESI) mode. The elemental analyses were performed using a Perkin-Elmer 2400 analyzer (PerkinElmer, Norwalk, CT, USA). TLC was performed on Uniplates (silica gel) purchased from Analtech Co. (7558, Newark, DE, USA). All reactions were performed under an atmosphere of nitrogen unless specified. Dry dichloromethane, benzene and pyridine were obtained by distillation from $\mathrm{CaH}_{2}$. Dry THF was obtained by distillation from $\mathrm{Na}$ and benzophenone immediately prior to use.

( \pm )-1-( $t$-Butyldimethylsilanyloxy)-pentan-3-ol (6). To a solution of $5(2.5 \mathrm{~g}, 13.27 \mathrm{mmol})$ in dry THF $(25 \mathrm{~mL})$ was slowly added ethylmagnesium bromide $(15.9 \mathrm{~mL}, 1.0 \mathrm{M}$ solution in THF) at $-20{ }^{\circ} \mathrm{C}$ and stirred $5 \mathrm{~h}$ at $0{ }^{\circ} \mathrm{C}$. Saturated $\mathrm{NH}_{4} \mathrm{Cl}$ solution $(16 \mathrm{~mL})$ was added to the mixture, which was slowly warmed to rt. The mixture was further diluted with water $(80 \mathrm{~mL})$ and extracted with EtOAc $(2 \times 80 \mathrm{~mL})$. The combined organic layer was washed with brine, dried over anhydrous $\mathrm{MgSO}_{4}$, filtered, and evaporated under reduced pressure. The residue was purified by silica gel column chromatography (EtOAc/hexane, 1:6) to give $6(2.58 \mathrm{~g}, 89 \%)$ as a colorless oil: ${ }^{1} \mathrm{H} \mathrm{NMR}\left(\mathrm{CDCl}_{3}\right.$, $300 \mathrm{MHz}) \delta 3.71(\mathrm{t}, J=6.8 \mathrm{~Hz}, 2 \mathrm{H}), 3.32(\mathrm{~m}, 2 \mathrm{H}), 1.45(\mathrm{~m}, 2 \mathrm{H})$, $0.97(\mathrm{t}, J=6.9 \mathrm{~Hz}, 3 \mathrm{H}), 0.81(\mathrm{~s}, 9 \mathrm{H}), 0.01(\mathrm{~s}, 6 \mathrm{H}) ;{ }^{13} \mathrm{C} \mathrm{NMR}$ $\left(\mathrm{CDCl}_{3}, 75 \mathrm{MHz}\right) \delta 70.7,58.8,42.5,32.1,25.5,18.5,9.8,-5.4$.

1-(t-Butyldimethylsilanyloxy)-pentan-3-one (7). To a solution of compound $6(1.39 \mathrm{~g}, 6.4 \mathrm{mmol})$ in $\mathrm{CH}_{2} \mathrm{Cl}_{2}(50 \mathrm{~mL}), 4 \AA$ molecular sieves $(3.75 \mathrm{~g})$ and PCC (3.45 g, $16.05 \mathrm{mmol})$ were added slowly at $0{ }^{\circ} \mathrm{C}$, and stirred overnight at room temperature. To the mixture, excess diethyl ether $(400 \mathrm{~mL})$ was then added. The mixture was stirred vigorously for $3 \mathrm{~h}$ at the same temperature, and the resulting solid was filtered through a short silica gel column. The filtrate was concentrated under vacuum and the residue was purified by silica gel column chromatography (EtOAc/hexane, 1:15) to give compound $7(1.08 \mathrm{~g}, 78 \%)$ as a colorless oil: ${ }^{1} \mathrm{H} \mathrm{NMR}\left(\mathrm{CDCl}_{3}, 300 \mathrm{MHz}\right) \delta 4.09(\mathrm{t}, J=7.0 \mathrm{~Hz}$, $2 \mathrm{H}), 2.68(\mathrm{t}, J=7.0 \mathrm{~Hz}, 2 \mathrm{H}), 2.52(\mathrm{q}, J=7.2 \mathrm{~Hz}, 2 \mathrm{H}), 1.09(\mathrm{t}$, $J=7.2 \mathrm{~Hz}, 3 \mathrm{H}), 0.82(\mathrm{~s}, 9 \mathrm{H}), 0.01(\mathrm{~s}, 6 \mathrm{H}) ;{ }^{13} \mathrm{C} \mathrm{NMR}\left(\mathrm{CDCl}_{3}\right.$, $75 \mathrm{MHz}) \delta 206.7,60.4,34.3,25.6,18.3,10.5,-5.5$.
( \pm )-5-(t-Butyldimethylsilanyloxy)-3-ethyl-pent-1-en-3-ol (8). To a solution of $7(2.5 \mathrm{~g}, 11.55 \mathrm{mmol})$ in dry THF $(25 \mathrm{~mL})$ was slowly added vinylmagnesium bromide $(12.7 \mathrm{~mL}, 1.0 \mathrm{M}$ solution in THF) at $-20{ }^{\circ} \mathrm{C}$ and stirred $4 \mathrm{~h}$ at $0{ }^{\circ} \mathrm{C}$. Saturated $\mathrm{NH}_{4} \mathrm{Cl}$ solution $(17 \mathrm{~mL}$ ) was added to the mixture, which was slowly warmed to rt. The mixture was further diluted with water $(80 \mathrm{~mL})$ and extracted with EtOAc $(2 \times 80 \mathrm{~mL})$. The combined organic layer was washed with brine, dried over anhydrous $\mathrm{MgSO}_{4}$, filtered, and evaporated under reduced pressure. The residue was purified by silica gel column chromatography (EtOAc/ hexane, $1: 12)$ to give $8(2.25 \mathrm{~g}, 80 \%)$ as a colorless oil: ${ }^{1} \mathrm{H} \mathrm{NMR}$ $\left(\mathrm{CDCl}_{3}, 300 \mathrm{MHz}\right) \delta 5.89(\mathrm{~m}, 1 \mathrm{H}), 5.21-5.12(\mathrm{~m}, 2 \mathrm{H}), 3.82(\mathrm{t}$, $J=6.2 \mathrm{~Hz}, 2 \mathrm{H}), 1.70(\mathrm{t}, J=6.3 \mathrm{~Hz}, 2 \mathrm{H}), 1.45(\mathrm{q}, J=6.9 \mathrm{~Hz}, 2 \mathrm{H})$, $0.97(\mathrm{t}, J=6.8 \mathrm{~Hz}, 3 \mathrm{H}), 0.81(\mathrm{~s}, 9 \mathrm{H}), 0.01(\mathrm{~s}, 6 \mathrm{H}) ;{ }^{13} \mathrm{C} \mathrm{NMR}$ $\left(\mathrm{CDCl}_{3}, 75 \mathrm{MHz}\right) \delta 144.1,112.7,71.5,58.9,46.2,34.6,25.5$, 18.5, 11.8,-5.6; Anal. Calc. for $\mathrm{C}_{13} \mathrm{H}_{28} \mathrm{O}_{2} \mathrm{Si}$ : C, 63.87; H, 11.55; Found: C, 63.92; H, 11.52 .

( \pm )-t-Butyl-[3-ethyl-3-(4-methoxybenzyloxy)-pent-4-enyloxy]-dimethylsilane (9). $\mathrm{NaH}$ (60\% in mineral oil, $207 \mathrm{mg}$, $83.21 \mathrm{mmol}$ ) was added portion-wise to a solution of alcohol 8 (3.5 g, $14.31 \mathrm{mmol})$ and $p$-methoxybenzyl chloride $(2.46 \mathrm{~g}$, $15.74 \mathrm{mmol})$ in DMF $(20 \mathrm{~mL})$ at $0{ }^{\circ} \mathrm{C}$. The reaction mixture was stirred at room temperature overnight. The solvent was concentrated under reduced pressure and the residue was quenched with $\mathrm{H}_{2} \mathrm{O}$ followed by extraction with diethyl ether. The organic layer was washed with brine, dried over anhydrous $\mathrm{MgSO}_{4}$, and concentrated under reduced pressure. The residue was purified by silica gel column chromatography (EtOAc/hexane, 1:15) to give $9(4.33 \mathrm{~g}, 83 \%)$ as a colorless oil. ${ }^{1} \mathrm{H} \mathrm{NMR}\left(\mathrm{CDCl}_{3}, 300\right.$ MHz) $\delta 7.27$ (m, 2H), $6.88(\mathrm{~m}, 2 \mathrm{H}), 5.95-5.86(\mathrm{~m}, 1 \mathrm{H}), 5.15-$ $5.03(\mathrm{~m}, 2 \mathrm{H}), 4.49(\mathrm{~s}, 2 \mathrm{H}), 3.81(\mathrm{~s}, 3 \mathrm{H}), 3.73(\mathrm{t}, J=6.8 \mathrm{~Hz}, 2 \mathrm{H})$, $1.65(\mathrm{t}, J=6.7 \mathrm{~Hz}, 2 \mathrm{H}), 1.46(\mathrm{~m}, 2 \mathrm{H}), 0.95(\mathrm{t}, J=6.9 \mathrm{~Hz}, 3 \mathrm{H})$, $0.82(\mathrm{~s}, 9 \mathrm{H}), 0.02(\mathrm{~s}, 6 \mathrm{H}) ;{ }^{13} \mathrm{C} \mathrm{NMR}\left(\mathrm{CDCl}_{3}, 75 \mathrm{MHz}\right) \delta 159.3$, 143.7, 136.2, 131.5, 128.7, 117.6, 112.9, 72.4, 71.2, 69.2, 58.3, 55.6, 43.1, 32.6, 25.3, 18.4, 10.6, -5.6; Anal. Calc. for $\mathrm{C}_{21} \mathrm{H}_{36}$ $\mathrm{O}_{3} \mathrm{Si}: \mathrm{C}, 69.18 ; \mathrm{H}, 9.95$; Found: C, 69.23; H, 9.89.

( \pm )-3-Ethyl-3-(4-methoxybenzyloxy)-pent-4-en-1-ol (10). To a solution of $9(300 \mathrm{mg}, 0.823 \mathrm{mmol})$ in THF $(8.0 \mathrm{~mL})$, TBAF $(1.0 \mathrm{~mL}, 1.0 \mathrm{M}$ solution in THF $)$ was added at $0{ }^{\circ} \mathrm{C}$. The mixture was stirred for $6 \mathrm{~h}$ at room temperature and concentrated in vacuo. The residue was purified by silica gel column chromatography (Hexane/EtOAc, 5:1) to give 10 (183 mg, $89 \%):{ }^{1} \mathrm{H} \mathrm{NMR}\left(\mathrm{CDCl}_{3}, 300 \mathrm{MHz}\right) \delta 7.29(\mathrm{~m}, 2 \mathrm{H}), 6.89(\mathrm{~m}$, $2 \mathrm{H}), 5.94-5.85(\mathrm{~m}, 1 \mathrm{H}), 5.20-5.09(\mathrm{~m}, 2 \mathrm{H}), 4.50(\mathrm{~s}, 2 \mathrm{H}), 3.83$ $(\mathrm{s}, 3 \mathrm{H}), 3.75(\mathrm{t}, J=6.7 \mathrm{~Hz}, 2 \mathrm{H}), 1.66(\mathrm{t}, J=6.8 \mathrm{~Hz}, 2 \mathrm{H}), 1.45$ $(\mathrm{q}, J=6.8 \mathrm{~Hz}, 2 \mathrm{H}), 0.97(\mathrm{t}, J=6.8 \mathrm{~Hz}, 3 \mathrm{H}) ;{ }^{13} \mathrm{C} \mathrm{NMR}\left(\mathrm{CDCl}_{3}\right.$, $75 \mathrm{MHz}) \delta 159.7,143.8,138.5,132.1,129.0,119.2,115.1,71.9$, 71.0, 68.3, 58.8, 54.8, 44.7, 33.1; Anal. Calc. for $\mathrm{C}_{15} \mathrm{H}_{22} \mathrm{O}_{3}$ : C, 71.97; H, 8.86; Found: C, 71.94; H, 8.91.

3-Ethyl-3-(4-methoxybenzyloxy)-pent-4-enal (11). To a stirred solution of oxalyl chloride $(212 \mathrm{mg}, 1.67 \mathrm{mmol})$ in $\mathrm{CH}_{2} \mathrm{Cl}_{2}(10 \mathrm{~mL})$ was added a solution of DMSO (195 mg, 2.5 $\mathrm{mmol})$ in $\mathrm{CH}_{2} \mathrm{Cl}_{2}(5.0 \mathrm{~mL})$ dropwise at $-78{ }^{\circ} \mathrm{C}$. The resulting solution was stirred at $-78{ }^{\circ} \mathrm{C}$ for $10 \mathrm{~min}$, and a solution of alcohol 10 (210 mg, $0.839 \mathrm{mmol})$ in $\mathrm{CH}_{2} \mathrm{Cl}_{2}(10 \mathrm{~mL})$ was added dropwise. The mixture was stirred at $-78{ }^{\circ} \mathrm{C}$ for $30 \mathrm{~min}$ and TEA (507 mg, $5.01 \mathrm{mmol}$ ) was added. The resulting mixture was warmed to $0{ }^{\circ} \mathrm{C}$ and stirred for $30 \mathrm{~min}$. $\mathrm{H}_{2} \mathrm{O}(15 \mathrm{~mL})$ was added, 
and the solution was stirred at room temperature for $30 \mathrm{~min}$. The mixture was diluted with water $(120 \mathrm{~mL})$ and then extracted with EtOAc $(2 \times 120 \mathrm{~mL})$. The combined organic layer was washed with brine, dried over anhydrous $\mathrm{MgSO}_{4}$, and filtered. The filtrate was concentrated under reduced pressure and the residue was purified by silica gel column chromatography (EtOAc/ hexane, 1:15) to give aldehyde compound 11 (189 mg, 91\%) as a colorless oil: ${ }^{1} \mathrm{H} \mathrm{NMR}\left(\mathrm{CDCl}_{3}, 300 \mathrm{MHz}\right) \delta 9.82(\mathrm{~s}, 1 \mathrm{H})$, $7.30(\mathrm{~m}, 2 \mathrm{H}), 6.91(\mathrm{~m}, 2 \mathrm{H}), 5.93-5.85(\mathrm{~m}, 1 \mathrm{H}), 5.20-5.11(\mathrm{~m}$, $2 \mathrm{H}), 4.49(\mathrm{~s}, 2 \mathrm{H}), 3.78(\mathrm{~s}, 3 \mathrm{H}), 2.55(\mathrm{dd}, J=10.0 .8 .2 \mathrm{~Hz}, 2 \mathrm{H})$, $1.47(\mathrm{q}, J=6.9 \mathrm{~Hz}, 2 \mathrm{H}), 0.98(\mathrm{t}, J=6.8 \mathrm{~Hz}, 3 \mathrm{H}) ;{ }^{13} \mathrm{C} \mathrm{NMR}$ $\left(\mathrm{CDCl}_{3}, 75 \mathrm{MHz}\right) \delta 205,160.0,144.1,138.3,133.6,128.9,121.6$, 114.1, 71.9, 69.1, 55.1, 32.6, 9.8.

(rel)-(3R and 3S,5S)-5-Ethyl-5-(4-methoxybenzyloxy)-hepta-1,6-dien-3-ol (12). Divinyl analogue 12 was prepared from aldehyde $\mathbf{1 1}$ using the similar procedure as described for $\mathbf{8}$ as a diastereomeric mixture: yield $87 \% ;{ }^{1} \mathrm{H} \mathrm{NMR}\left(\mathrm{CDCl}_{3}, 300\right.$ $\mathrm{MHz}) \delta$ 7.29-7.27 (m, 2H), 6.89 (m, 2H), 5.96-5.83 (m, 2H), 5.16-4.99 (m, 4H), $4.59(\mathrm{~s}, 2 \mathrm{H}), 3.89(\mathrm{~m}, 1 \mathrm{H}), 3.78(\mathrm{~s}, 3 \mathrm{H})$, $1.65-1.58(\mathrm{~m}, 2 \mathrm{H}), 1.48(\mathrm{~m}, 2 \mathrm{H}), 0.97(\mathrm{t}, J=6.9 \mathrm{~Hz}, 3 \mathrm{H}) ;{ }^{13} \mathrm{C}$ $\mathrm{NMR}\left(\mathrm{CDCl}_{3}, 75 \mathrm{MHz}\right) \delta 159.6,143.7,141.2,137.5,134.0$, 127.5, 121.9, 113.1, 112.4, 71.5, 69.6, 68.2, 57.5, 47.0, 33.3, 9.6.

(rel)-(1S,4S)-4-Ethyl-4-(4-methoxybenzyloxy)-cyclopent2-enol (13a) and (rel)-(1R,4S)-4-ethyl-4-(4-methoxybenzyloxy)-cyclopent-2-enol (13b). To a solution of 12 (205 mg, 0.74 $\mathrm{mmol})$ in dry methylene chloride $(6 \mathrm{~mL})$ was added 2 nd generation Grubbs catalyst $(38.0 \mathrm{mg} 0.0452 \mathrm{mmol})$. The reaction mixture was refluxed overnight and cooled to room temperature. The mixture was concentrated in vacuo, and the residue was purified by silica gel column chromatography (EtOAc/hexane, $1: 12)$ to give cyclopentenol 13a $(71.6 \mathrm{mg}, 39 \%)$ and $\mathbf{1 3 b}(73.5$ $\mathrm{mg}, 40 \%$ ).

Data for 13a: ${ }^{1} \mathrm{H} \mathrm{NMR}\left(\mathrm{CDCl}_{3}, 300 \mathrm{MHz}\right) \delta 7.31(\mathrm{~m}, 2 \mathrm{H})$, $6.88(\mathrm{~m}, 2 \mathrm{H}), 5.65(\mathrm{~d}, J=5.4 \mathrm{~Hz}, 1 \mathrm{H}), 5.36(\mathrm{~m}, 1 \mathrm{H}), 4.68(\mathrm{~s}$, 2H), $4.08(\mathrm{~m}, 1 \mathrm{H}), 3.79(\mathrm{~s}, 3 \mathrm{H}), 2.16(\mathrm{dd}, J=13.4 .8 .8 \mathrm{~Hz}$, 1H), $2.02(\mathrm{dd}, J=13.4,6.8 \mathrm{~Hz}, 1 \mathrm{H}), 1.52(\mathrm{~m}, 2 \mathrm{H}), 0.97(\mathrm{t}, J=$ $7.0 \mathrm{~Hz}, 3 \mathrm{H}) ;{ }^{13} \mathrm{C} \mathrm{NMR}\left(\mathrm{CDCl}_{3}, 75 \mathrm{MHz}\right) \delta 159.5,139.3,136.6$, 134.4, 130.5, 129.6, 118.4, 78.9, 71.8, 68.6, 57.0, 42.8, 32.6, 9.4; Anal. Calc. for $\mathrm{C}_{15} \mathrm{H}_{20} \mathrm{O}_{3}$ : C, 72.55; $\mathrm{H}, 8.12$; Found: $\mathrm{C}, 72.58$; H, 8.15.

Data for 13b: ${ }^{1} \mathrm{H}$ NMR $\left(\mathrm{CDCl}_{3}, 300 \mathrm{MHz}\right) \delta 7.29(\mathrm{~m}, 2 \mathrm{H})$, $6.91(\mathrm{~m}, 2 \mathrm{H}), 5.58(\mathrm{~d}, J=5.4 \mathrm{~Hz}, 1 \mathrm{H}), 5.36(\mathrm{dd}, J=5.3,4.2$ $\mathrm{Hz}, 1 \mathrm{H}), 4.69$ (s, 2H), 4.03 (dd, $J=6.0,4.8 \mathrm{~Hz}, 1 \mathrm{H}), 3.81(\mathrm{~s}, 3 \mathrm{H})$, $2.19(\mathrm{dd}, J=13.6 .8 .2 \mathrm{~Hz}, 1 \mathrm{H}), 2.06(\mathrm{dd}, J=13.5,7.6 \mathrm{~Hz}, 1 \mathrm{H})$, $1.49(\mathrm{~m}, 2 \mathrm{H}), 0.98(\mathrm{t}, J=6.9 \mathrm{~Hz}, 3 \mathrm{H}) ;{ }^{13} \mathrm{C} \mathrm{NMR}\left(\mathrm{CDCl}_{3}, 75\right.$ $\mathrm{MHz}) \delta 159.7,138.8,135.7,130.4,130.5,129.6,117.4,79.1$, 72.3, 69.3, 56.7, 43.2, 33.4, 9.6; Anal. Calc. for $\mathrm{C}_{15} \mathrm{H}_{20} \mathrm{O}_{3}$ : C, 72.55; H, 8.12; Found: C, 72.49; H, 8.08.

(rel)-(1'R,4'S)-9-[4-Ethyl-(4-methoxybenzyloxy)-cyclopent2-enyl]-6-chloropurine (14). To a solution containing compound 13b (112 mg, $0.45 \mathrm{mmol}$ ), triphenylphosphine (415 mg, $1.584 \mathrm{mmol}$ ), and 6-chloropurine (139 $\mathrm{mg}, 0.90 \mathrm{mmol}$ ) in anhydrous THF (7.0 mL), diisopropyl azodicarboxylate (DIAD) (182 mg, $0.90 \mathrm{mmol}$ ) was added dropwise at $-20{ }^{\circ} \mathrm{C}$ for $30 \mathrm{~min}$ under nitrogen. The reaction mixture was stirred for $3 \mathrm{~h}$ at $-20{ }^{\circ} \mathrm{C}$ under nitrogen and further stirred overnight at rt. The solvent was concentrated under reduced pressure and the residue was purified by silica gel column chromatography (EtOAc/hexane,
2:1) to give compound 14 (74 mg, $43 \%$ ): $\mathrm{mp} 156-158^{\circ} \mathrm{C}$; UV $(\mathrm{MeOH}) \lambda_{\max } 264.5 \mathrm{~nm} ;{ }^{1} \mathrm{H} \mathrm{NMR}\left(\mathrm{CDCl}_{3}, 300 \mathrm{MHz}\right) \delta 8.72(\mathrm{~s}$, $1 \mathrm{H}), 8.44$ (s, 1H), 7.30 (m, 2H), 6.90-6.87 (m, 2H), 5.64 (d, $J=$ $5.3 \mathrm{~Hz}, 1 \mathrm{H}), 5.35$ (dd, $J=5.2,4.2 \mathrm{~Hz}, 1 \mathrm{H}), 4.64$ (s, 2H), 4.43 $(\mathrm{m}, 1 \mathrm{H}), 3.80(\mathrm{~s}, 3 \mathrm{H}), 2.21(\mathrm{dd}, J=13.5 .8 .4 \mathrm{~Hz}, 1 \mathrm{H}), 2.04(\mathrm{dd}$, $J=13.6,7.2 \mathrm{~Hz}, 1 \mathrm{H}), 1.53(\mathrm{~m}, 2 \mathrm{H}), 0.96(\mathrm{t}, J=6.9 \mathrm{~Hz}, 3 \mathrm{H})$; ${ }^{13} \mathrm{C} \mathrm{NMR}\left(\mathrm{CDCl}_{3}, 75 \mathrm{MHz}\right) \delta 159.6,152.6,151.3,147.9,141.5$, 137.5, 135.3, 133.4, 117.7, 81.3, 70.9, 57.1, 54.6, 38.7, 32.6, 9.7; Anal. Calc. for $\mathrm{C}_{20} \mathrm{H}_{21} \mathrm{ClN}_{4} \mathrm{O}_{2}$ (+ 0.5 EtOAc): C, 61.60; H, 5.87; N, 13.06; Found: C, 61.56; H, 5.90; N, 13.11 .

(rel)-(1'R,4'S)-9-(4-Ethyl-4-hydroxycyclopent-2-enyl)-6chloropurine (15). To a solution of compound 14 (435 mg, $1.13 \mathrm{mmol})$ in $\mathrm{CH}_{2} \mathrm{Cl}_{2} / \mathrm{H}_{2} \mathrm{O}$ mixture $(10 \mathrm{~mL}, 10: 1 \mathrm{v} / \mathrm{v})$ was added DDQ (280 mg, $1.24 \mathrm{mmol})$ and the mixture was stirred for $4 \mathrm{~h}$ at room temperature. Saturated $\mathrm{NaHCO}_{3}(1.5 \mathrm{~mL})$ was added to quench the reaction and further diluted with water $(80$ $\mathrm{mL})$. The mixture was extracted with $\mathrm{CH}_{2} \mathrm{Cl}_{2}(3 \times 80 \mathrm{~mL})$ and the combined organic layer was dried over anhydrous $\mathrm{MgSO}_{4}$, and filtered. The filtrate was concentrated under reduced pressure and the residue was purified by silica gel column chromatography (EtOAc/hexane/MeOH, 4:1:0.04) to give compound 15 (200 mg, 67\%): mp $169-171{ }^{\circ} \mathrm{C}$; UV (MeOH) $\lambda_{\max }$ $264.0 \mathrm{~nm} ;{ }^{1} \mathrm{H}$ NMR (DMSO- $\left.d_{6}, 300 \mathrm{MHz}\right) \delta 8.75(\mathrm{~s}, 1 \mathrm{H}), 8.46$ $(\mathrm{s}, 1 \mathrm{H}), 5.59(\mathrm{~d}, J=5.2 \mathrm{~Hz}, 1 \mathrm{H}), 5.36(\mathrm{~m}, 1 \mathrm{H}), 5.09(\mathrm{~s}, 1 \mathrm{H})$, $4.50(\mathrm{~m}, 1 \mathrm{H}), 2.25(\mathrm{dd}, J=13.6 .8 .5 \mathrm{~Hz}, 1 \mathrm{H}), 2.06(\mathrm{dd}, J=13.5$, $7.4 \mathrm{~Hz}, 1 \mathrm{H}), 1.51(\mathrm{~m}, 2 \mathrm{H}), 0.98(\mathrm{t}, J=7.0 \mathrm{~Hz}, 3 \mathrm{H}) ;{ }^{13} \mathrm{C} \mathrm{NMR}$ (DMSO- $\left.d_{6}, 75 \mathrm{MHz}\right) \delta 152.3,151.6,150.5,148.2,138.5,136.6$, 132.4, 76.8, 52.9, 40.3, 34.3, 9.3; Anal. Calc. for $\mathrm{C}_{12} \mathrm{H}_{13} \mathrm{ClN}_{4} \mathrm{O}$ : C, 54.45; H, 4.95; N, 21.17; Found: C, 54.51; H, 4.91; N, 21.21.

(rel)-(1'R,4'S)-Diethyl [9-(4-Hydroxy-4-ethylcyclopent-2en-1-yl)-6-chloropurine] methylphosphonate (16). Both $\mathrm{LiO} t$ $\mathrm{Bu}(2.084 \mathrm{~mL}$ of $0.5 \mathrm{M}$ solution in THF, $1.042 \mathrm{mmol})$ and a solution of diethyl phosphonomethyltriflate (313 mg, 1.042 $\mathrm{mmol}$ ) in $10.0 \mathrm{~mL}$ of THF were slowly added to a solution of the 6-chloropurine analogue $15(138 \mathrm{mg}, 0.521 \mathrm{mmol})$ in $5.0 \mathrm{~mL}$ of THF at $0{ }^{\circ} \mathrm{C}$ and stirred overnight at $\mathrm{rt}$ under anhydrous conditions. The mixture was quenched by adding saturated $\mathrm{NH}_{4} \mathrm{Cl}$ solution $(3 \mathrm{~mL})$ and further diluted with additional $\mathrm{H}_{2} \mathrm{O}(80 \mathrm{~mL})$. The aqueous layer was extracted with EtOAc $(3 \times 80 \mathrm{~mL})$. The combined organic layer was dried over anhydrous $\mathrm{MgSO}_{4}$ and concentrated in vacuo. The residue was purified by silica gel column chromatography $(\mathrm{MeOH} / \mathrm{Hexane} / \mathrm{EtOAc}, 0.05: 4: 1)$ to give 16 (153 mg, 71\%): $\mathrm{mp} 142-144^{\circ} \mathrm{C}$; $\mathrm{UV}(\mathrm{MeOH}) \lambda_{\max } 265.0$ $\mathrm{nm} ;{ }^{1} \mathrm{H}$ NMR (DMSO- $\left.d_{6}, 300 \mathrm{MHz}\right) \delta 8.74(\mathrm{~s}, 1 \mathrm{H}), 8.48(\mathrm{~s}$, $1 \mathrm{H}), 5.62(\mathrm{~d}, J=5.4 \mathrm{~Hz}, 1 \mathrm{H}), 5.35(\mathrm{~m}, 1 \mathrm{H}), 4.51(\mathrm{~m}, 1 \mathrm{H}), 4.18$ $(\mathrm{m}, 4 \mathrm{H}), 4.09(\mathrm{~d}, J=8.0 \mathrm{~Hz}, 2 \mathrm{H}), 2.28(\mathrm{dd}, J=13.6 .8 .7 \mathrm{~Hz}, 1 \mathrm{H})$, 2.04 (dd, $J=13.6,7.5 \mathrm{~Hz}, 1 \mathrm{H}), 1.52(\mathrm{~m}, 2 \mathrm{H}), 1.36(\mathrm{~m} 6 \mathrm{H}), 0.98$ $(\mathrm{m}, 3 \mathrm{H}) ;{ }^{13} \mathrm{C}$ NMR (DMSO- $\left.d_{6}, 75 \mathrm{MHz}\right) \delta 152.3,151.9,149.3$, 146.6, 145.7, 136.5, 132.6, 81.8, 66.5, 65.2, 63.6, 54.1, 38.2, 32.5, 16.8, 9.6; Anal. Calc. for $\mathrm{C}_{17} \mathrm{H}_{24} \mathrm{ClN}_{4} \mathrm{O}_{4} \mathrm{P}(+1.0 \mathrm{MeOH})$ : C, 48.38; H, 6.31; N, 12.54; Found: C, 48.44; H, 6.27; N, 12.49.

(rel)-(1'R,4'S)-Diethyl [9-(4-Hydroxy-1,4-dimethylcyclopent-2-en-1-yl)-adenine] methylphosphonate (17). A solution of $16(132 \mathrm{mg}, 0.318 \mathrm{mmol})$ in saturated methanolic ammonia $(8 \mathrm{~mL})$ was stirred at $70{ }^{\circ} \mathrm{C}$ in a steel bomb, and the volatiles were evaporated. The residue was purified by silica gel column chromatography $\left(\mathrm{MeOH} / \mathrm{CH}_{2} \mathrm{Cl}_{2}, 1: 10\right)$ to give 17 (74 mg, 59\%): mp $171-173{ }^{\circ} \mathrm{C}$; UV (MeOH) $\lambda_{\max } 260.5 \mathrm{~nm} ;{ }^{1} \mathrm{H}$ NMR 
(DMSO- $\left.d_{6}, 300 \mathrm{MHz}\right) \delta 8.32(\mathrm{~s}, 1 \mathrm{H}), 8.12(\mathrm{~s}, 1 \mathrm{H}), 7.17$ (br s, $2 \mathrm{H}), 5.67(\mathrm{~d}, J=5.2 \mathrm{~Hz}, 1 \mathrm{H}), 5.31(\mathrm{dd}, J=5.3,4.2 \mathrm{~Hz}, 1 \mathrm{H})$, $4.52(\mathrm{~m}, 1 \mathrm{H}), 4.17(\mathrm{~m}, 4 \mathrm{H}), 4.05(\mathrm{~d}, J=8.2 \mathrm{~Hz}, 2 \mathrm{H}), 2.26(\mathrm{dd}$, $J=13.5 .8 .8 \mathrm{~Hz}, 1 \mathrm{H}), 2.05(\mathrm{~m}, 1 \mathrm{H}), 1.51(\mathrm{~m}, 2 \mathrm{H}), 1.34(\mathrm{~m} 6 \mathrm{H})$, $0.98(\mathrm{t}, J=6.9 \mathrm{~Hz}, 3 \mathrm{H}) ;{ }^{13} \mathrm{C}$ NMR (DMSO- $\left.d_{6}, 75 \mathrm{MHz}\right) \delta 155.0$, 153.3, 144.2, 137.7, 134.2, 131.8, 119.2, 82.4, 67.1, 66.0, 64.2, 54.5, 39.1, 33.7, 17.2, 9.9; Anal. Calc. for $\mathrm{C}_{17} \mathrm{H}_{26} \mathrm{~N}_{5} \mathrm{O}_{4} \mathrm{P}(+0.5$ $\mathrm{MeOH}):$ C, 51.09; H, 6.86; N, 17.02; Found: C, 51.15; H, 6.92; $\mathrm{N}, 16.98$.

(rel)-(1'R,4'S)-[9-(4-Ethylcyclopenten-1-yl)-adenine]-4methylphosphonic acid (18). To a solution of the phosphonate 17 (125 mg, $0.316 \mathrm{mmol})$ in anhydrous $\mathrm{CH}_{3} \mathrm{CN}(10 \mathrm{~mL})$ and 2,6-lutidine $(0.8 \mathrm{~mL})$ was added trimethylsilyl bromide $(483$ $\mathrm{mg}, 3.16 \mathrm{mmol})$. The mixture was heated overnight at $60{ }^{\circ} \mathrm{C}$ and then concentrated under reduced pressure. The residue was partitioned between $\mathrm{CH}_{2} \mathrm{Cl}_{2}(70 \mathrm{~mL})$ and distilled clean water $(60 \mathrm{~mL})$. The aqueous layer was washed out with $\mathrm{CH}_{2} \mathrm{Cl}_{2}$ two times and then freeze-dried to give phosphonic acid 18 (73 mg, $68 \%$ ) in a yellowish form: UV $\left(\mathrm{H}_{2} \mathrm{O}\right) \lambda_{\max } 260.5 \mathrm{~nm} ;{ }^{1} \mathrm{H}$ NMR (DMSO-d, $300 \mathrm{MHz}) \delta 8.29(\mathrm{~s}, 1 \mathrm{H}), 8.11(\mathrm{~s}, 1 \mathrm{H}), 7.16(\mathrm{br} \mathrm{s}$, $2 \mathrm{H}), 5.64(\mathrm{~d}, J=5.3 \mathrm{~Hz}, 1 \mathrm{H}), 5.32(\mathrm{dd}, J=5.4,4.2 \mathrm{~Hz}, 1 \mathrm{H}), 4.50$ $(\mathrm{m}, 1 \mathrm{H}), 4.11(\mathrm{~d}, J=8.0 \mathrm{~Hz}, 2 \mathrm{H}), 2.29(\mathrm{~m}, 1 \mathrm{H}), 2.02(\mathrm{dd}, J=13.6$, $7.2 \mathrm{~Hz}, 1 \mathrm{H}), 1.52(\mathrm{~m}, 2 \mathrm{H}), 0.96(\mathrm{~m}, 3 \mathrm{H}) ;{ }^{13} \mathrm{C}$ NMR (DMSO- $d_{6}$, $75 \mathrm{MHz}) \delta 154.7,152.9,142.7,136.8,135.5,132.7,120.1$, 81.9, 65.2, 56.3, 38.7, 34.1, 9.6; Anal. Calc. for $\mathrm{C}_{13} \mathrm{H}_{18} \mathrm{~N}_{5} \mathrm{O}_{4} \mathrm{P}$ $\left(+2.0 \mathrm{H}_{2} \mathrm{O}\right): \mathrm{C}, 41.60 ; \mathrm{H}, 5.91 ; \mathrm{N}, 18.66$; Found: $\mathrm{C}, 41.56 ; \mathrm{H}$, $5.92 ; \mathrm{N}, 18.59$.

(rel)-(1'R,2'S,3'S,4'S)-Diethyl [9-(2,3-dihydroxy-4-ethylcyclopent-1-yl)-adenine]-4-methylphosphonate (19) and (rel)(1'R,2'R,3'R,4'S)-diethyl [9-(2,3-dihydroxy-4-ethyl-cyclopent1-yl)-adenine]-4-methylphosphonate (20). Compound 17 (158 $\mathrm{mg}, 0.4 \mathrm{mmol})$ was dissolved in a cosolvent system $(10 \mathrm{~mL})$ (acetone: $t$ - $\mathrm{BuOH}: \mathrm{H}_{2} \mathrm{O}=6: 1: 1$ ) along with 4-methylmorpholine $\mathrm{N}$-oxide ( $82 \mathrm{mg}, 0.8 \mathrm{mmol})$. Subsequently, $\mathrm{OsO}_{4}(0.19 \mathrm{~mL}, 0.03$ mmol, $4 \%$ wt $\%$ in $\mathrm{H}_{2} \mathrm{O}$ ) was added. The mixture was stirred overnight at $\mathrm{rt}$ and quenched with saturated $\mathrm{Na}_{2} \mathrm{SO}_{3}$ solution $(3 \mathrm{~mL})$. The resulting solid was removed by filtration through a pad of Celite, and filtrate was concentrated under reduced pressure. The residue was purified by silica gel column chromatography ( $\left.\mathrm{MeOH} / \mathrm{CH}_{2} \mathrm{Cl}_{2}, 1: 6\right)$ to give 19 (58 mg, 34\%) and 20 (49 mg, 29\%): Spectroscopical data for 19: ${ }^{1} \mathrm{H}$ NMR (DMSO$\left.d_{6}, 300 \mathrm{MHz}\right) \delta 8.30(\mathrm{~s}, 1 \mathrm{H}), 8.13(\mathrm{~s}, 1 \mathrm{H}), 7.15$ (br s, 2H), 4.25 $(\mathrm{m}, 4 \mathrm{H}), 4.14(\mathrm{~d}, J=8.2 \mathrm{~Hz}, 2 \mathrm{H}), 3.75-3.68(\mathrm{~m}, 2 \mathrm{H}), 3.32(\mathrm{~m}$, $1 \mathrm{H}), 2.13(\mathrm{dd}, J=13.6,8.7 \mathrm{~Hz}, 1 \mathrm{H}), 1.94(\mathrm{dd}, J=13.6,7.2 \mathrm{~Hz}$, 1H), $1.48(\mathrm{~m}, 2 \mathrm{H}), 1.31(\mathrm{~m} 6 \mathrm{H}), 0.98(\mathrm{t}, J=6.9 \mathrm{~Hz}, 3 \mathrm{H}) ;{ }^{13} \mathrm{C}$ NMR (DMSO- $\left.d_{6}, 75 \mathrm{MHz}\right) \delta 154.6,153.2,147.7,138.4,119.3$, 78.4, 77.6, 69.1, 66.2, 65.1, 63.7, 48.6, 28.8, 26.4, 17.1, 9.9; Anal. Calc. for $\mathrm{C}_{17} \mathrm{H}_{28} \mathrm{~N}_{5} \mathrm{O}_{6} \mathrm{P}(+1.0 \mathrm{MeOH}): \mathrm{C}, 46.85 ; \mathrm{H}, 6.99$; N, 15.17; Found: C, 46.91; H, 7.05; N, 15.13.

Spectroscopical data for 20: ${ }^{1} \mathrm{H}$ NMR (DMSO- $d_{6}, 300 \mathrm{MHz}$ ) $\delta 8.34(\mathrm{~s}, 1 \mathrm{H}), 8.15$ (s, 1H), 7.17 (br s, 2H), 4.27(m, 4H), 4.12 $(\mathrm{d}, J=8.1 \mathrm{~Hz}, 2 \mathrm{H}), 3.76(\mathrm{dd}, J=6.4,5.2 \mathrm{~Hz}, 1 \mathrm{H}), 3.66(\mathrm{~d}, J=$ $6.0 \mathrm{~Hz}, 1 \mathrm{H}), 3.25(\mathrm{~m}, 1 \mathrm{H}), 2.15(\mathrm{dd}, J=13.7,8.8 \mathrm{~Hz}, 1 \mathrm{H}), 1.96$ (dd, $J=13.6,7.4 \mathrm{~Hz}, 1 \mathrm{H}), 1.50(\mathrm{~m}, 2 \mathrm{H}), 1.32(\mathrm{~m} 6 \mathrm{H}), 0.97(\mathrm{~m}$, $3 \mathrm{H}) ;{ }^{13} \mathrm{C}$ NMR (DMSO- $\left.d_{6}, 75 \mathrm{MHz}\right) \delta 154.5,151.7,146.8,134.6$, 120.7, 79.3, 76.2, 68.5, 65.8, 64.2, 63.0, 47.3, 30.6, 27.5, 16.8, 9.5; Anal. Calc. for $\mathrm{C}_{17} \mathrm{H}_{28} \mathrm{~N}_{5} \mathrm{O}_{6} \mathrm{P}(+1.0 \mathrm{MeOH}): \mathrm{C}, 46.85 ; \mathrm{H}$, 6.99; N, 15.17; Found: C, 46.79; H, 6.95; N, 15.20.
(rel)-(1'R,2'S,3'S,4'S)-[9-(2,3-Dihydroxy-4-ethylcyclopent1-yl)] adenine]-4-methylphosphonic acid (21). The final adenosine phosphonic acid $\mathbf{2 1}$ was synthesized from 19 using a similar procedure described for $\mathbf{1 8}$ as a formy solid: yield $60 \%$; $\mathrm{UV}\left(\mathrm{H}_{2} \mathrm{O}\right) \lambda_{\max } 261.0 \mathrm{~nm} ;{ }^{1} \mathrm{H}$ NMR (DMSO- $\left.d_{6}, 300 \mathrm{MHz}\right) \delta 8.29$ $(\mathrm{s}, 1 \mathrm{H}), 8.14(\mathrm{~s}, 1 \mathrm{H}), 3.98(\mathrm{~d}, J=8.0 \mathrm{~Hz}, 2 \mathrm{H}), 3.78(\mathrm{~m}, 1 \mathrm{H}), 3.68$ $(\mathrm{d}, J=6.1 \mathrm{~Hz}, 1 \mathrm{H}), 3.22(\mathrm{~m}, 1 \mathrm{H}), 2.13(\mathrm{dd}, J=13.8,8.7 \mathrm{~Hz}$, $1 \mathrm{H}), 1.95(\mathrm{dd}, J=13.8,7.6 \mathrm{~Hz}, 1 \mathrm{H}), 1.51(\mathrm{~m}, 2 \mathrm{H}), 0.95(\mathrm{~m}, 3 \mathrm{H})$; ${ }^{13} \mathrm{C}$ NMR (DMSO- $\left.d_{6}, 75 \mathrm{MHz}\right) \delta 154.9,152.5,147.4,132.8$, 119.6, 78.6, 77.3, 67.8, 64.9, 63.1, 48.1, 31.4, 28.8, 10.0; Anal. Calc. for $\mathrm{C}_{13} \mathrm{H}_{20} \mathrm{~N}_{5} \mathrm{O}_{6} \mathrm{P}\left(+3.0 \mathrm{H}_{2} \mathrm{O}\right): \mathrm{C}, 36.54 ; \mathrm{H}, 6.13 ; \mathrm{N}, 16.39$; Found: C, 36.49; H, 6.08; N, 16.44.

Acknowledgments. This work was supported by Research Grant of Chosun University (2008).

\section{References}

1. Maag, H.; Rydzewski, R. M.; McRoberts, M. J.; Crawford-Ruth, D.; Verheyden, J. P.; Prisbe, E. J. J. Med. Chem. 1992, 35, 1440.

2. Sugimoto, I.; Shuto, S.; Mori, S.; Shigeta, S.; Matsuda, A. Bioorg. Med. Chem. Lett. 1999, 9, 385.

3. Boojamra, C. G.; Parrish, J. P.; Sperandio, D.; Gao, Y.; Petrakovsky, O. V.; Lee, S. K.; Markevich, D. Y.; Vela, J. E.; Laflamme, G.; Chen, J. M.; Ray, A. S.; Barron, A. C.; Sparacino, M. L.; Desai, M. C.; Kim, C. U.; Cihlar, T.; Mackman, R. L. Bioorg. Med. Chem. 2009, 17, 1739

4. (a) Mackman, R. L.; Boojamra, C. G.; Prasad, V.; Zhang, L.; Lin, K. Y.; Petrakovsky, O.; Babusis, D.; Chen, J.; Douglas, J.; Grant, D.; Hui, H. C.; Kim, C. U.; Markevitch, D. Y.; Vela, J.; Ray, A.; Cihlar, T. Bioorg. Med. Chem. Lett. 2007, 17, 6785. (b) Ray, A. S.; Vela, J. E.; Boojamra, C. G.; Zhang, L.; Hui, H.; Callebaut, C.; Stray, K.; Lin, K. Y.; Gao, Y.; Mackman, R. L.; Cihlar, T. Antimicrob. Agents Chemother. 2008, 52, 648.

5. (a) Ray, A. S.; Vela, J. E.; Boojamra, C. G.; Zhang, L.; Hui, H.; Callebaut, C.; Stray, K.; Lin, K. Y.; Gao, Y.; Mackman, R. L.; Cihlar, T. Antimicrob. Agents Chemother. 2008, 52, 648. (b) Boojamra, C. G.; Mackman, R. L.; Markevitch, D. Y.; Prasad, V.; Ray, A. S.; Douglas, J.; Grant, D.; Kim, C. U.; Cihlar, T. Bioorg. Med. Chem. Lett. 2008, 18, 1120. (c) Mackman, R. L.; Lin, K. Y.; Boojamra, C. G.; Hui, H.; Douglas, J.; Grant, D.; Petrakovsky, O.; Prasad, V.; Ray, A. S.; Cihlar, T. Bioorg. Med. Chem. Lett. 2008, 18, 1116.

6. Kim, C. U.; Luh, B. Y.; Misco, P. F.; Bronson, J. J.; Hitchcock, M. J.; Ghazzouli, I.; Martin, J. C. J. Med. Chem. 1990, 33, 1207.

7. Wu, T.; Froeyen, M.; Kempeneers, V.; Pannecouque, C.; Wang, J.; Busson, R.; De Clercq, E.; Herdewijn, P. J. Am. Chem. Soc. 2005, 127, 5056 .

8. Kim, C. U.; Luh, B. Y.; Martin, J. C. J. Org. Chem. 1991, 56, 2642.

9. Stoeckler, J. D.; Cambor, C.; Parks, R. E., Jr. Biochemistry 1980, $19,102$.

10. Heapy, A. M.; Bramble, M. A. Tetrahedron 2010, 66, 5424.

11. (a) Jeong, L. S.; Lee, J. A. Antiviral Chem. Chemother. 2004, 15, 235. (b) Amblard, F.; Nolan, S. P.; Agrofoglio, L. A. Tetrahedron 2005, 61, 7067.

12. (a) Liu, L. J.; Kim, S. W.; Lee, W.; Hong, J. H. Bull. Korean Chem. Soc. 2009, 30, 2989; (b) Li, H.; Kim, S. W.; Hong, J. H. Bull. Korean Chem. Soc. 2010, 31, 2180

13. Oh, H. S.; Kang, H. Y. Tetrahedron 2010, 66, 4307

14. (a) Phillion, D. P.; Andrew, S. S. Tetrahedron Lett. 1986, 27, 1477. (b) Xu, Y.; Flavin, M. T.; Xu, Z.-Q. J. Org. Chem. 1996, 61, 7697.

15. Hocková, D.; Holý, A.; Masojídková, M.; Keough, D. T.; De Jersey, J.; Guddat, L. W. Bioorg. Med. Chem. 2009, 17, 6218.

16. Koh, Y. H.; Shim, J. H.; Wu, J. Z.; Zhong, W.; Hong, Z.; Girardet, J. L. J. Med. Chem. 2005, 48, 2867.

17. Trost, B. M.; Kuo, G. H.; Benneche, T. J. Am. Chem. Soc. 1988, 110,621

18. Lian, L. J.; Yoo, J. C.; Hong, J. H. Nucleos. Nucleot. Nucl. 2009 , 28,150 . 